Published in final edited form as:

Exp Gerontol. 2013 December ; 48(12): 1395-1401. doi:10.1016/j.exger.2013.09.005.

\title{
Morbidity risks among older adults with pre-existing age-related diseases
}

\author{
Igor Akushevich, $\mathrm{PhD}^{1}$, Julia Kravchenko, MD, $\mathrm{PhD}^{2}$, Svetlana Ukraintseva, $\mathrm{PhD}^{1}$, \\ Konstantin Arbeev, PhD ${ }^{1}$, Alexander Kulminski, PhD ${ }^{1}$, and Anatoliy I. Yashin, PhD, Sc. ${ }^{1}$ \\ ${ }^{1}$ Center for Population Health and Aging, Duke University, Durham, NC 27708 \\ 2Department of Surgery, Duke University Medical Center, Duke University, Durham, NC 27705
}

\section{Abstract}

Multi-morbidity is common among older adults; however, for many aging-related diseases there is no information for U.S. elderly population on how earlier-manifested disease affects the risk of another disease manifested later during patient's lifetime. Quantitative evaluation of risks of cancer and non-cancer diseases for older adults with pre-existing conditions is performed using the Surveillance, Epidemiology, and End Results (SEER) Registry data linked to the Medicare Files of Service Use (MFSU). Using the SEER-Medicare data containing individual records for 2,154,598 individuals, we empirically evaluated age patterns of incidence of age-associated diseases diagnosed after the onset of earlier manifested disease and compared these patterns with those in general population. Individual medical histories were reconstructed using information on diagnoses coded in MFSU, dates of medical services/procedures, and Medicare enrollment/ disenrollment. More than threefold increase of subsequent diseases risk was observed for 15 disease pairs, majority of them were i) diseases of the same organ and/or system (e.g., Parkinson disease for patients with Alzheimer disease, $\mathrm{HR}=3.77$, kidney cancer for patients with renal failure, $\mathrm{HR}=3.28$ ) or ii) disease pairs with primary diseases being fast-progressive cancers (i.e., lung, kidney, and pancreas), e.g., ulcer $(\mathrm{HR}=4.68)$ and melanoma $(\mathrm{HR}=4.15)$ for patients with pancreatic cancer. Lower risk of subsequent disease was registered for 20 disease pairs, mostly among patients with Alzheimer's or Parkinson's disease, e.g., decreased lung cancer risk among patients with Alzheimer's ( $\mathrm{HR}=0.64)$ and Parkinson's $(\mathrm{HR}=0.60)$ disease. Synergistic and antagonistic dependences in geriatric disease risks were observed among US elderly confirming known and detecting new associations of wide spectrum of age-associated diseases. The results can be used in optimization of screening, prevention and treatment strategies of chronic diseases among U.S. elderly population.

\section{Keywords}

Medicare; chronic disease onset; dependent risks; comorbidity; aging; geriatric disease

\footnotetext{
(c) 2013 Elsevier Inc. All rights reserved.

Corresponding Author: Igor Akushevich, PhD, Center for Population Health and Aging, Duke University, 002 Trent Dr., Durham, NC 27708, Phone: (919) 668-2715, Fax: (919) 684-3861, igor.akushevich@ duke.edu.

Publisher's Disclaimer: This is a PDF file of an unedited manuscript that has been accepted for publication. As a service to our customers we are providing this early version of the manuscript. The manuscript will undergo copyediting, typesetting, and review of the resulting proof before it is published in its final citable form. Please note that during the production process errors may be discovered which could affect the content, and all legal disclaimers that apply to the journal pertain.
} 


\section{INTRODUCTION}

Approximately $96 \%$ of Medicare expenditures were accounted for by the 2/3 of beneficiaries with multiple chronic conditions (Anderson, Solutions, \& Foundation, 2004). At advanced ages, people are at their highest multi-morbidity risks that also lead to growing medical expenditures. The risks of different diseases may be not independent: disease that has occurred earlier can increase or decrease the probability of occurrence of the disease that will be diagnosed later. To evaluate these relationships, two types of disease risks should be distinguished: conditional (calculated for cohort of the patients who have the disease of interest) and unconditional (calculated for the general population). The conditional risk reflects the interrelations between an earlier occurred (pre-existing) disease and a subsequent disease (manifested later in time), while the unconditional risk of disease occurrence does not depend on the presence of other diseases in the patient. The conditional risk of a certain disease could be higher (in this case pre-existing disease is a risk factor for this disease) or lower (in this case pre-existing condition possesses certain protective effect against this disease) than the unconditional risk. Understanding and evaluation of relationships between the aging-associated diseases risks are important for both public health and clinical practice -i.e., at the population and individual patient's levels. The detailed information on dependent risks helps to develop the prognoses of population morbidity (including disease incidence and prevalence) and mortality with a better accuracy; specifically, the effects of pre-existing diseases on risks of later-in-life diagnosed diseases can be incorporated into disease incidence prognosis and into the health forecasting models for specific population groups. Also, understanding the possible underlying causes of observed phenomena of dependence between disease risks (e.g., the effects of treatment of earlier occurred disease that can increase or decrease the risk of later occurred disease, shared behavioral risks, pleotropic effects of genes) can be used for developing new preventive strategies and therapeutic approaches. Recently, several pairs of diseases whose risks were influenced by the presence of another pre-existing disease have been described: e.g., cancer and circulatory diseases, cancer and Parkinson's disease, stroke and Alzheimer's disease, cancer and diabetes, and asthma and cancer (Tabarés-Seisdedos et al., 2011; Ukraintseva et al., 2010).

However, systematic information about disease dependence is largely lacking. We considered 21 diseases representing various systems of human organism and evaluated the conditional and unconditional disease risks using empirical methods and more formal approaches based on the proportional hazard models. Specific attentions were paid to pairs of diseases of the same organ, the same system, and diseases for which a pre-existent condition decreased or substantially increased their risks.

The Medicare-based datasets provide an excellent, underexplored opportunity for systematic investigation of the mutual dependence of aging-associated diseases risks at the national level. The progress in this area became possible after development and validation of a computational approach allowed for identifying the date of onset of cancer and non-cancer diseases for Medicare beneficiaries using longitudinal information from Medicare Files of Service Use (MFSU) (Akushevich et al., 2012; Yashin et al., 2010). The Surveillance, Epidemiology, and End Results (SEER) Registry data linked to MFSU is one of the largest sources of such information for cancer and non-cancer diseases. Accordingly, the SEERMedicare date are used in analyses presented this paper.

\section{Data and Methods}

SEER-Medicare data-The expanded SEER registry covers approximately $26 \%$ of the U.S. population. In total, the Medicare records for 2,154,598 individuals are available in SEER-Medicare including individuals i) with diagnosed cancers of breast $(\mathrm{n}=353,285)$, colon $(n=222,659)$, lung $(n=342,961)$, prostate $(n=448,410)$ and skin melanoma 
( $\mathrm{n}=101,123)$; and ii) from a random 5\% sample of Medicare beneficiaries residing in the SEER areas who had none of the above mentioned cancers. For the majority of patients, we have continuous records of Medicare services use from 1991 (or from the time the person has passed the age of 65 after 1990) until his/her death. A small fraction of individuals (e.g., new patients diagnosed with cancer in 2003-2005) has Medicare records from 1998.

Medicare records are available for each institutional (MedPAR, outpatient, hospice, or home health agency HHA) and non-institutional (Carrier-Physician-Supplier and Durable Medical Equipment Providers) claim type.

Computation of dates of onsets and disease rates-Twenty one diseases of various systems were selected for analyses based on the following selection criteria: i) most common cancers (lung, colon, female breast, and prostate) and cancers with increasing incidence (skin melanoma, kidney, pancreatic); ii) highly prevalent diseases of cardio(myocardial infarction, angina pectoris, heart failure) and cerebrovascular (stroke) systems, respiratory system (chronic obstructive pulmonary disease (COPD), asthma), and kidney and gastrointestinal tract (chronic renal disease/failure, ulcer); iii) highly prevalent (Parkinson's and Alzheimer's) neurodegenerative diseases; iv) highly prevalent endocrine disease (diabetes) or disorder with growing prevalence and public health concern (goiter); v) highly prevalent autoimmune disease with high disability (rheumatoid arthritis); and vi) trauma/injury associated with high medical costs and disabilities (hip fracture). The ages at disease onsets were reconstructed from the MSUF data using the scheme described in detail in Akushevich, et al. (2012). In brief, individual medical histories for each selected disease were reconstructed from MSUF by combining all records with their respective ICD-9 codes (listed in Supplementary Table 1). Then, a special computational procedure was applied for individuals with the histories of considered disease to separate the incident and prevalent cases and to identify the age at the disease onset. An individual was treated as having a disease onset when he/she had two records (occurred during 0.3 year interval) with respective ICD-9 code as a primary diagnosis in one of four Medicare sources: inpatient care, outpatient care, physician services, and skilled nursing facilities. The first of these two records was interpreted as a preliminary diagnosis, and the second record provided a confirmation of the diagnosis. The date of the disease onset was the date of the first record.

Identified dates of disease onsets for all diseases were combined into the individual medical history containing information about the date of entry into the follow-up, the dates of disease onsets (if any), and the date of, and survival status at, the end of individual follow-up. This information was used for evaluation of age-patterns of unconditional and conditional disease incidences. Unconditional disease incidence was evaluated for each disease for the total population, and the conditional rates were evaluated for cohorts of individuals after another disease onset. The proportional hazard model of disease incidence with the age as the follow-up variable was used to estimate the hazard ratios of occurrence vs. non-occurrence of the disease with earlier onset (i.e., pre-existing disease). The model was applied for all individuals in the dataset. Since individual follow-up could start from different ages (e.g., because of relocation to the SEER areas after age 65), we used the proportional hazard model with left truncation. The dependent variable is the occurrence of the pre-existence condition. The binary indicator function is the respective time-dependent covariate. It equals 0 or 1 for time periods of individual follow-up before or after the onset of the earlier occurred disease.

\section{RESULTS}

In total, 420 pairs of diseases were analyzed. For each pair, we calculated the age patterns of the unconditional incidence rates of the diseases, conditional rates of later-in-life diagnosed disease for individuals after onset of the earlier diagnosed disease, and the hazard ratios of 
onset of later occurred disease in the presence (or absence) of the pre-existing disease. The most interesting results are presented in Figures 1 and 2, and all sets of plots are given in Supplementary Figure 1. The three evaluated quantities, i.e., conditional and unconditional rates and hazard ratios, are presented in each cell of these Figures. We focused on identifying three groups of interrelations between the studied diseases: i) diseases whose risk became much higher when patients had certain pre-existed (earlier diagnosed) disease (Figure 1); ii) disease whose risk became lower than in the general population when patients had certain pre-existing conditions (so called "trade-off" effect between earlier and later occurred diseases) (Figure 2); and iii) diseases for which "the two-tail" effects were observed: i.e., when the effects are significant for both orders of disease precedence; both effects can be direct (any of the disease from a disease pair increases the risk of another disease), inverse (any of the disease from a disease pair decreases the risk of another disease), or controversial (one disease increases the risk of another, but another disease decreases the risk of the second disease from a considered disease pair).

Fifteen pairs of diseases with high (above 3.0) and significant HRs $(\mathrm{p}<0.001)$ were identified (Figure 1). Three types of interrelations were detected. The first one comprises diseases pairs when the later diagnosed diseases (both cancers and non-cancers) occurred in patients who had earlier diagnosed cancers that are characterized by quick progression (i.e., cancers of lung, kidney, and pancreas): cancers of breast $(\mathrm{HR}=3.3)$ and kidney $(\mathrm{HR}=4.6)$ in lung cancer patients; cancers of lung $(\mathrm{HR}=3.1)$ and pancreas $(\mathrm{HR}=3.6)$, and chronic kidney disease $(\mathrm{HR}=3.3)$ among kidney cancer patients; and cancers of lung $(\mathrm{HR}=4.9)$, colon $(\mathrm{HR}=3.4)$ and breast $(\mathrm{HR}=3.8)$, melanoma $(\mathrm{HR}=4.2)$, and peptic ulcer $(\mathrm{HR}=4.7)$ among the patients with pancreas cancer. The second type of interrelations was when the later occurred disease was of the same organ/system: e.g., manifestation of Parkinson's disease after Alzheimer's disease (HR=3.8), angina pectoris after myocardial infarction $(\mathrm{HR}=3.3)$, asthma after COPD (excluding asthma) $(\mathrm{HR}=3.1)$, and kidney cancer after chronic renal disease $(\mathrm{HR}=3.3)$. Finally, the third type of interrelations was the type containing one disease pair not described by the above mentioned two types: manifestation of chronic renal disease in patients with heart failure $(\mathrm{HR}=3.3)$.

Eighteen disease pairs representing the "trade-off" effects with $\mathrm{HR}<0.9$ and $\mathrm{p}<0.05$ were selected (Figure 2). Four types of the interrelations can be distinguished. The first group was observed for Parkinson's disease: patients with this disease had lower risk of lung $(\mathrm{HR}=0.60)$, colon $(\mathrm{HR}=0.57)$, breast $(\mathrm{HR}=0.65)$, and prostate $(\mathrm{HR}=0.67)$ cancers, and of angina pectoris $(\mathrm{HR}=0.74)$. The second group was detected for Alzheimer's disease: these patients had very low risk of melanoma $(H R=0.08)$ and pancreatic cancer $(H R=0.10)$, and lower risks of cancers of lung $(\mathrm{HR}=0.64)$ and breast $(\mathrm{HR}=0.54)$, angina pectoris $(\mathrm{HR}=0.49)$, asthma $(\mathrm{HR}=0.68)$, arthritis $(\mathrm{HR}=0.52)$, and goiter $(\mathrm{HR}=0.36)$. The third group involves COPD: its risk was lower among patients with breast $(\mathrm{HR}=0.86)$ and prostate $(\mathrm{HR}=0.80)$ cancers. And, the fourth group included a triad of such diseases as breast cancer, angina pectoris, and hip fracture: breast cancer risk was lower among patients with angina pectoris $(\mathrm{HR}=0.79)$ or hip fracture $(\mathrm{HR}=0.82)$, and angina pectoris risk was lower in patients who had a hip fracture $(\mathrm{HR}=0.78)$. Besides these findings, some trade-offs which have been reported in other studies were also observed: e.g., a decreased risk of prostate cancer among patients with diabetes $(\mathrm{HR}=0.91, \mathrm{p}=0.05)$ and decreased colon cancer risk in patients with arthritis $(\mathrm{HR}=0.77, \mathrm{p}=0.056)$.

Only four pairs of diseases (selected using $\mathrm{HR}<0.95$ and $\mathrm{p}<0.35$ ) had the reduced risk of the later diagnosed disease in both orders of precedence including breast cancer and angina pectoris $\left(\mathrm{HR}=0.79\right.$ and $0.91^{*}$, the first $\mathrm{HR}$ value corresponds to the situation when the disease that is listed second in the pair was pre-existing, the asterisk means a significant level $0.05<\mathrm{p}<0.35)$, melanoma and Alzheimer disease $\left(\mathrm{HR}=0.08\right.$ and $\left.0.67^{*}\right)$, asthma and 
Alzheimer disease $(\mathrm{HR}=0.68$ and $0.92 *)$, and kidney cancer and Parkinson disease $\left(\mathrm{HR}=0.63^{*}\right.$ and $\left.0.45^{*}\right)$.

In general, a majority of disease pairs with increased risk of the later diagnosed disease in both orders of precedence were those in which both the pre-existing and later occurred diseases were cancers, and also when both diseases were of the same organ. We selected twelve disease pairs with $H R>2$ and $p<0.05$. For the group with two cancers, these pairs were: lung cancer-colon cancer $(\mathrm{HR}=2.62$ and 2.53), lung-cancer-kidney cancer $(\mathrm{HR}=3.11$ and 4.62), lung cancer-pancreas cancer $(\mathrm{HR}=4.94$ and 2.95), colon cancerpancreas cancer $(H R=3.35$ and 2.79), and kidney cancer-pancreas cancer $(H R=3.93$ and 3.61). For diseases within the same organ/system, these pairs were: lung cancer-COPD $(\mathrm{HR}=2.53$ and 2.24), Parkinson's disease-Alzheimer's disease $(\mathrm{HR}=3.77$ and 2.03), myocardial infarction - heart failure $(\mathrm{HR}=2.09$ and 2.43), and chronic renal disease-kidney cancer $(\mathrm{HR}=3.64$ and 3.28). The three remaining pairs were myocardial infarction-chronic renal disease ( $\mathrm{HR}=2.26$ and 2.43), heart failure-chronic renal disease ( $\mathrm{HR}=2.08$ and 3.32), and diabetes-pancreatic cancer $(\mathrm{HR}=2.11$ and 2.17$)$.

Eight disease pairs had the opposite effects depending on the diseases order (i.e., diseases diagnosed earlier vs. later in life): lung cancer-arthritis $(\mathrm{HR}=1.32$ and $0.72 *)$, pancreatic cancer-prostate cancer $\left(\mathrm{HR}=1.53\right.$ and $\left.0.45^{*}\right)$, pancreatic cancer-asthma $(\mathrm{HR}=1.66$ and $\left.0.29^{*}\right)$, diabetes - asthma $(\mathrm{HR}=1.30$ and 0.90$)$, Parkinson disease - angina pectoris $(\mathrm{HR}=1.24$ and 0.74$)$, goiter-COPD $\left(\mathrm{HR}=1.33\right.$ and $\left.0.84^{*}\right)$, ulcer-diabetes $(\mathrm{HR}=1.33$ and $0.94 *)$, hip fracture - diabetes $(\mathrm{HR}=1.22$ and $0.91 *)$. Disease pair selection criteria were $H R<0.95$ and $p<0.35$ for decreased disease risks effects and $H R>1.2$ and $p<0.05$ for increased disease risks effects.

\section{DISCUSSION AND CONCLUSION}

In this paper we investigated how the occurrence of an aging-associated disease influences the chance of occurrence of another disease later in life in the US elderly population. Identifying such relationships is extremely important since they indicate that the development of seemingly unrelated cancer and non-cancer diseases may involve common biological mechanisms and/or risk factors. Some of found interrelations between risks confirmed the associations from other studies, e.g., reduced risk of prostate cancer among the patients with diabetes (Kasper \& Giovannucci, 2006), reduced risk of colon cancer among rheumatoid arthritis patients (Cibere et al., 2005; Thomas et al., 2000), and reduced risk of malignancies among Alzheimer's disease patients (Driver et al., 2012) (see Figure 2). There are few studies focused on analysis of interrelations between diseases, e.g., Hidalgo et al. (2009) studied correlations between comorbid diseases in the U.S. elderly population by using a Phenotypic Disease Network and showed that some diseases were highly connected and could be more lethal while the others were barely connected at all, and these connections differed by race and gender. To the best of our knowledge, the majority of interrelations between pre-existing and later occurred disease were firstly evaluated for the U.S. elderly patients in our study. One example is the decreased risk of breast cancer for hip fracture patients (Figure 2) compared to the general population; partly it could be explained by the low post-menopausal estrogen levels which has an opposite effect on risks of these two diseases (Fuhrman et al., 2012; Hannan et al., 2010; Karim et al., 2011; Rod et al., 2009). Another example is the increased risk of breast cancer evaluated for patients with goiter; the risk of goiter among breast cancer patients was also significant (see Figure 3). This is in agreement with the results of clinical and epidemiological studies that suggested important roles of iodine and triiodothyronine in thyroid functioning and breast cancer risk (Cann, van Netten, \& van Netten, 2000; Tosovic et al., 2010); however, some studies do not support interactions between thyroid disorders and increased risk of breast cancer (Simon et 
al., 2002). Several other examples of new associations that were evaluated in our study are described below. Broadly, the associations among disease risks can be explained by a higher or lower probability of using specific diagnostic procedures among patients who already had certain chronic diseases, by shared biological mechanisms, or by both factors. Indeed, there is a chance that a clinician could administer certain diagnostic procedures differently for patients with a specific medical history. That could explain, at least partly, a lower risks of later occurred diseases in patients with a history of specific diseases (e.g., among the patients with severe pre-existing diseases that may lead to under-diagnosis of asymptomatic stages of cancer). However, a systematic nature of the majority of associations obtained in our study cannot be entirely explained by this hypothesis. For example, while patients with Alzheimer's disease had a lower malignancy risk possibly because of the lower chance that malignancy would be diagnosed, the risks for myocardial infarction, renal diseases, and ulcer among Alzheimer's patients did not differ from the general population, and risk of stroke, hip fracture, heart failure (slightly), diabetes (slightly) and COPD (in older age group) were higher than in the general population (Supplementary Figure 1). Therefore, the alternative hypothesis about the role of treatment, shared risk factors, and genetics in dependence in disease risks could also be suggested.

We observed almost four-fold increased risk of Parkinson's disease among Alzheimer's patients. In addition these diseases had similar interrelations with other diseases: e.g., both were negatively correlated with asthma risk. Recently, it has been shown that Alzheimer's and Parkinson's diseases may share a common genetic background, such as variations in NEDD9 and APOE genes (Li et al., 2008; Pankratz et al., 2006). Association between the risks of Parkinson's and Alzheimer's diseases might be, to some extent, due to miscoding of Parkinson-induced dementia as Alzheimer's. However, different spectrums of associations with other disease do not confirm this hypothesis: e.g., melanoma risk was higher among the patients with Parkinson's disease, but was lower among Alzheimer's patients (see Figure 4). For Parkinson's disease, levodopa (widely used for its treatment) could increase melanoma risk (Fiala et al., 2003). Increased melanoma risk among Parkinson's patients could be also explained by the common genetic profile of these disease, as well as being attributed to a confounding role of social class (e.g., through an inverse relationship with tobacco smoking) (Zanetti et al., 2006). In contrast, melanoma risk among Alzheimer's patients tends to be lower than in the general population; one of the possible factors is that levodopa is not a mainstream treatment for Alzheimer's disease (Ou et al., 2012). Interesting, that skin nonmelanoma risk was increased among Alzheimer's patients (Panelos \& Massi, 2009). Arthritis also had opposite association with these two neurological disorders: its risk was lower among Alzheimer's disease but not among Parkinson's disease patients (see Figure 4). Published data on these associations are sparse and controversial (Heyman et al., 2004), with the most of the studies focused on opposite correlation such as lower risk of Alzheimer's disease among patients with arthritis (e.g., due to the use of non-steroid anti-inflammatory drugs) (Szekely et al., 2004).

The observed antagonistic relationships (the trade-offs) between cancer and Alzheimer's or Parkinson's diseases are in agreement with our previous studies (Ukraintseva, et al., 2010; Yashin et al., 2009) and allows to suggest an inverse association between the carcinogenesis and neurodegeneration. These associations could be explained by biological mechanisms of diseases: e.g., through an inappropriate activation and deregulation of the cell cycle involving Pin1 and p53 (Driver, et al., 2012). However, while we observed lower risk of all cancers among Alzheimer's patients, the risk of Alzheimer's disease among cancer patients did not change or was just slightly reduced, depending on cancer site. That could be due to the dualistic impact of chemo- and radiation therapy on patients' cognitive function: cancer treatment suppresses an inflammation and/or block the entry into the cell cycle thus decreasing Alzheimer's disease risk, but at the same time cancer treatment may cause a 
cognitive impairment in cancer patients (Driver, et al., 2012). The final impact from these two effects depends on more powerful influence and can also result into "zero" impact (Driver, et al., 2012). In addition the hypothesis that a shared biological background may play the role in obtained associations can be supported by the findings of a common genetic background for different diseases, i.e., in the form of pleotropy (Sivakumaran et al., 2011). Furthermore, recent studies also documented the genetic trade-offs: i.e., when the same alleles confer the risks of several diseases (e.g., CVD, Alzheimer's disease) but protect against the others (e.g., cancer) (Kulminski et al., 2011; van Heemst et al., 2005). The carriers of the e4 allele have generally lower CRP levels (Chasman, Kozlowski, Zee, Kwiatkowski, \& Ridker, 2006), which, in their turn, are associated with reduced cancer risk and better prognosis (Koukourakis et al., 2009; Nikiteas et al., 2005). That does not contradict our findings that the degree of reduction between risks of cancers and Alzheimer's disease is different in both orders of disease precedence. The highest incidence for cancers and Alzheimer's diseases occur at different ages and the efficiency of the same genes can be different at different ages (Ilveskoski et al., 1999; Kulminski et al., 2013).

Interestingly, most of the earlier manifested diseases increased the risk of renal disease. The risk of chronic renal disease for patients with heart failure and kidney cancer is shown in Figure 1. Several other examples are shown in Figure 5. This may be because kidney is very vulnerable to damages included by adverse effects of treatment of other diseases. Considering this, no wonder renal disease is one of the major causes of death in the elderly. Kidney protection may become a priority in disease prevention and strategies aimed at increasing an overall survival.

In our study, the risk of asthma was lower among the patients with Alzheimer's and Parkinson's diseases, carcinomas of prostate, kidney, and pancreas, and skin melanoma. However, lung, pancreatic, and (less prominent) colon cancers were more frequent among asthma patients than in the general population. Several examples of respective age patterns and HR estimates are given in Figures 1 and 2. The existence of inverse associations between allergic disorders including asthma and cancer risk remains controversial: significant inverse associations have been reported for history of asthma and hay fever and overall cancer mortality ( $\mathrm{RR}=0.88,95 \% \mathrm{CI} 0.83-0.93$ ), while asthma itself was associated with lower leukemia risk only (Turner et al., 2005). An increased risk of lung cancer among patients with asthma observed in our study is in agreement with some other studies: it could be due to the shared risk factors such as smoking, certain occupational exposures (Boffetta et al., 2002), and chronic bronchiolar inflammation (especially, it is important for nonsmokers) (Santillanet al., 2003). Also, asthma treatment may reduce an "increased alert" of the immune system to cancer cells (Friedman et al., 1998).

Surprisingly, few diseases substantially influenced the risk of diabetes: it may be a true independence, but may be as well a hidden trade-off: e.g., changes in dietary patterns and decreasing obesity risk due to the lifestyle changes caused by earlier diagnosed disease, as well as certain medications use (such as immunodepressants) may decrease a subsequent risk of diabetes (Solomon et al., 2011). However, some of the widely used medicine such as antidepressants and statins may result in increased risk of diabetes (Rajpathak et al., 2009; Rubin et al., 2008). Among cancers, increased risk of colon, kidney, and pancreatic carcinomas was observed in our study for patients with the history of diabetes (see Figure 6). This is in agreement with other studies in which the role of insulin-like growth factor I (IGF-I), insulin therapy, and hyperinsulinemia have been suggested to promote tumor cells growth (Atchison et al., 2012; Flood et al., 2010; Yang et al., 2004). Increased colon cancer risk, in a part, could be also explained by a slower bowel transit in people with diabetes which increases an exposure to carcinogens; also, an increased production of carcinogenic bile acids may be the issue (Coughlin \& Giovannucci, 2012). Our data showed that 
pancreatic cancer and diabetes both increased each other risks (Figure 6). While the link between diabetes and pancreatic cancer has long been suggested (Farrow \& Davis, 1990), however, it is still controversial whether diabetes is risk factor for or consequence of pancreatic cancer (Wang et al., 2006).

Generally, the effect of dependence between two pathologies diminishes with advancing age. This could be because senescence itself becomes a leading risk factor of death in the oldest old, so that at the very old age it matters less if a person is healthy or sick, because its vulnerability to death is high anyway due to a dramatic decline in the body's overall resistance to stresses attributed to aging (Ukraintseva \& Yashin, 2003). Respectively, a relative risk of death from specific causes diminishes with aging.

\section{Study limitations}

A unifying approach to the identification of disease onset and the calculation of the incidence rates was used for all considered diseases. On the one hand this is a limitation of the paper. On the other hand this assumption allows us to have a common picture and to compare mutual disease effects. The date of onset of a chronic disease is not defined with the same precision as mortality and there is always certain arbitrariness in defining the date of onset. The date of onset can be identified using information collected in MFSU with specific assumptions which outline the specific calculation algorithm. The computational approaches of different complexity simply correspond to different definitions of disease incidence. Moreover, different strategies can be used in clinical practice for different diseases. For example, the diagnostic criteria of different heart studies reviewed in the Appendix of ref. (NIH/NHLBI, 2006) do not allow us to unambiguously conclude that our choice exactly corresponds to those made in these studies. Thus, certain arbitrariness in defining the date of onset was used for constructing a unified definition of date of onset appropriate for population studies.

In our analysis death was treated as a censoring event independent of the second disease onset. In the general case the risks of death and disease onsets could be dependent and thus, survival could influence the chance of occurrence of another disease later in life. The survival effect could be analyzed using the sensitivity analysis (e.g., as in Yashin et al., 2010) or a model of dependent competing risks (Yashin et al., 1986).

Although, in total 420 disease pairs were analyzed and specific estimates could require an adjustment for multiple testing, applying the Bonferroni correction did not affect the conclusions about mutual disease effects: e.g., all HRs presented in Fig 1 had pvalue $<0.0001$ (except the pair of pancreas cancer and melanoma, $\mathrm{p}=0.0015$ ) which remains significant after the Bonferroni correction.

In summary, we developed an approach to evaluation of conditional disease risks using the information from MFSU. A unified computational approach applied to all considered diseases within the same analysis allowed us to create a unified view on the mutual interrelationships among the risks of cancer and non-cancer ageing-associated diseases. Direct and inverse dependences in geriatric disease risks were observed among US elderly confirming known and detecting new associations of a wide spectrum of diseases. The existence of inverse associations for the later-in-life diagnosed disease risk may provide important insights into disease mechanisms and new opportunities for disease prevention and therapy, allowing to focus on increase in healthy lifespan rather than concentrating the efforts on reduction of risk for each particular disease alone. The better understanding of the biological links between different diseases (or between the groups of diseases-etiological or organ-specific) can provide new therapeutic approaches for diseases with the shared pathological pathways. The Medicare data files are nationally representative; therefore, the 
observed mutual dependence of the studied diseases onset could be used for improvement of current models forecasting future morbidity and mortality, for planning medical expenditures, and for optimization of screening and preventive strategies among elderly population with multi-morbid conditions.

\section{Supplementary Material}

Refer to Web version on PubMed Central for supplementary material.

\section{References}

Akushevich I, Kravchenko J, Ukraintseva S, Arbeev K, Yashin AI. Age patterns of incidence of geriatric disease in the u.s. Elderly population: medicare-based analysis. J Am Geriatr Soc. 2012; 60(2):323-327. [PubMed: 22283485]

Anderson, G. Solutions JHUPf \& Foundation RWJ. Chronic conditions: Making the case for ongoing care. Johns Hopkins University; 2004.

Atchison EA, Gridley G, Carreon JD, Leitzmann MF, McGlynn KA. Risk of cancer in a large cohort of US veterans with diabetes. International Journal of Cancer. 2010; 128(3):635-643.

Boffetta P, Ye W, Boman G, Nyren O. Lung cancer risk in a population-based cohort of patients hospitalized for asthma in Sweden. European Respiratory Journal. 2002; 19(1):127-133. [PubMed: 11843311]

Cann SA, van Netten JP, van Netten C. Hypothesis: iodine, selenium and the development of breast cancer. Cancer Causes and Control. 2000; 11(2):121-127. [PubMed: 10710195]

Chasman D, Kozlowski P, Zee R, Kwiatkowski D, Ridker P. Qualitative and quantitative effects of APOE genetic variation on plasma C-reactive protein, LDL-cholesterol, and apoE protein. Genes and immunity. 2006; 7(3):211-219. [PubMed: 16511556]

Cibere J, Sibley J, Haga M. Rheumatoid arthritis and the risk of malignancy. Arthritis \& Rheumatism. 2005; 40(9):1580-1586. [PubMed: 9324011]

Coughlin SS, Giovannucci EL. Diabetes and Cancer. Diabetes Chronic Complications. 2012:294.

Driver JA, Beiser A, Au R, Kreger BE, Splansky GL, Kurth T, et al. Inverse association between cancer and Alzheimer's disease: results from the Framingham Heart Study. BMJ. 2012; 344

Farrow D, Davis S. Risk of pancreatic cancer in relation to medical history and the use of tobacco, alcohol and coffee. International Journal of Cancer. 1990; 45(5):816-820.

Fiala KH, Whetteckey J, Manyam BV. Malignant melanoma and levodopa in Parkinson's disease: causality or coincidence? Parkinsonism \& related disorders. 2003; 9(6):321-327. [PubMed: 12853231]

Flood A, Strayer L, Schairer C, Schatzkin A. Diabetes and risk of incident colorectal cancer in a prospective cohort of women. Cancer Causes and Control. 2010; 21(8):1277-1284. [PubMed: 20383575]

Friedman GD, Coates AO, Potter JD, Slattery ML. Drugs and colon cancer. Pharmacoepidemiology and drug safety. 1998; 7(2):99-106. [PubMed: 15073733]

Fuhrman BJ, Schairer C, Gail MH, Boyd-Morin J, Xu X, Sue LY, et al. Estrogen metabolism and risk of breast cancer in postmenopausal women. J Natl Cancer Inst. 2012; 104(4):326-339. [PubMed: 22232133]

Hannan MT, Felson DT, Dawson-Hughes B, Tucker KL, Cupples LA, Wilson PW, et al. Risk factors for longitudinal bone loss in elderly men and women: the Framingham Osteoporosis Study. Journal of Bone and Mineral Research. 2010; 15(4):710-720. [PubMed: 10780863]

Heyman A, Wilkinson WE, Stafford JA, Helms MJ, Sigmon AH, Weinberg T. Alzheimer's disease: a study of epidemiological aspects. Annals of neurology. 2004; 15(4):335-341. [PubMed: 6742780]

Hidalgo CA, Blumm N, Barabási AL, Christakis NA. A dynamic network approach for the study of human phenotypes. PLoS computational biology. 2009; 5(4):e1000353. [PubMed: 19360091]

Ilveskoski E, Perola M, Lehtimäki T, Laippala P, Savolainen V, Pajarinen J, et al. Age-dependent association of apolipoprotein E genotype with coronary and aortic atherosclerosis in middle-aged men: an autopsy study. Circulation. 1999; 100(6):608-613. [PubMed: 10441097] 
Karim R, Dell RM, Greene DF, Mack WJ, Gallagher JC, Hodis HN. Hip fracture in postmenopausal women after cessation of hormone therapy: results from a prospective study in a large health management organization. Menopause. 2011; 18(11):1172. [PubMed: 21775911]

Kasper JS, Giovannucci E. A meta-analysis of diabetes mellitus and the risk of prostate cancer. Cancer Epidemiology Biomarkers \& Prevention. 2006; 15(11):2056-2062.

Koukourakis MI, Kambouromiti G, Pitsiava D, Tsousou P, Tsiarkatsi M, Kartalis G. Serum C-reactive protein (CRP) levels in cancer patients are linked with tumor burden and are reduced by antihypertensive medication. Inflammation. 2009; 32(3):169-175. [PubMed: 19373547]

Kulminski AM, Culminskaya I, Arbeev KG, Ukraintseva SV, Stallard E, Arbeeva L, et al. The role of lipid-related genes, aging-related processes, and environment in healthspan. Aging Cell. 2013

Kulminski AM, Culminskaya I, Ukraintseva SV, Arbeev KG, Arbeeva L, Wu D, et al. Trade-off in the effects of the apolipoprotein E polymorphism on the ages at onset of CVD and cancer influences human lifespan. Aging Cell. 2011; 10(3):533-541. [PubMed: 21332925]

Li Y, Grupe A, Rowland C, Holmans P, Segurado R, Abraham R, et al. Evidence that common variation in NEDD9 is associated with susceptibility to late-onset Alzheimer's and Parkinson's disease. Human molecular genetics. 2008; 17(5):759-767. [PubMed: 18063669]

NIH/NHLBI. Incidence and Prevalence: 2006 Chart Book on Cardiovascular and Lung Diseases. 2006. Retrieved June, 2010, from http://www.nhlbi.nih.gov/resources/docs/06a_ip_chtbk.pdf

Nikiteas NI, Tzanakis N, Gazouli M, Rallis G, Daniilidis K, Theodoropoulos G, et al. Serum IL-6, TNFa and CRP levels in Greek colorectal cancer patients: prognostic implications. World J Gastroenterol. 2005; 11:1639-1643. [PubMed: 15786541]

Ou SM, Lee YJ, Hu YW, Liu CJ, Chen TJ, Fuh JL, et al. Does Alzheimer's Disease Protect against Cancers? A Nationwide Population-Based Study. Neuroepidemiology. 2012; 40(1):42-49. [PubMed: 23075910]

Panelos J, Massi D. Emerging role of Notch signaling in epidermal differentiation and skin cancer. Cancer biology \& therapy. 2009; 8(21):1986-1993. [PubMed: 19783903]

Pankratz N, Byder L, Halter C, Rudolph A, Shults CW, Conneally PM, et al. Presence of an APOE4 allele results in significantly earlier onset of Parkinson's disease and a higher risk with dementia. Movement disorders. 2006; 21(1):45-49. [PubMed: 16116614]

Rajpathak SN, Kumbhani DJ, Crandall J, Barzilai N, Alderman M, Ridker PM. Statin therapy and risk of developing type 2 diabetes: a meta-analysis. Diabetes Care. 2009; 32(10):1924-1929. [PubMed: 19794004]

Rod NH, Hansen ÅM, Nielsen J, Schnohr P, Grønbæk M. Low-risk factor profile, estrogen levels, and breast cancer risk among postmenopausal women. International Journal of Cancer. 2009; 124(8): 1935-1940.

Rubin RR, Ma Y, Marrero DG, Peyrot M, Barrett-Connor EL, Kahn SE, et al. Elevated depression symptoms, antidepressant medicine use, and risk of developing diabetes during the diabetes prevention program. Diabetes Care. 2008; 31(3):420-426. [PubMed: 18071002]

Santillan AA, Camargo CA, Colditz GA. A meta-analysis of asthma and risk of lung cancer (United States). Cancer Causes and Control. 2003; 14(4):327-334. [PubMed: 12846363]

Simon MS, Tang MTC, Bernstein L, Norman SA, Weiss L, Burkman RT, et al. Do thyroid disorders increase the risk of breast cancer? Cancer Epidemiology Biomarkers \& Prevention. 2002; 11(12): 1574-1578.

Sivakumaran S, Agakov F, Theodoratou E, Prendergast JG, Zgaga L, Manolio T, et al. Abundant pleiotropy in human complex diseases and traits. The American Journal of Human Genetics. 2011; 89(5):607-618.

Solomon DH, Massarotti E, Garg R, Liu J, Canning C, Schneeweiss S. Association between diseasemodifying antirheumatic drugs and diabetes risk in patients with rheumatoid arthritis and psoriasis. JAMA. 2011; 305(24):2525. [PubMed: 21693740]

Szekely CA, Thorne JE, Zandi PP, Ek M, Messias E, Breitner JC, et al. Nonsteroidal antiinflammatory drugs for the prevention of Alzheimer's disease: a systematic review. Neuroepidemiology. 2004; 23(4):159-169. [PubMed: 15279021] 
Tabarés-Seisdedos R, Dumont N, Baudot A, Valderas JM, Climent J, Valencia A, et al. No paradox, no progress: inverse cancer comorbidity in people with other complex diseases. The lancet oncology. 2011

Thomas E, Brewster DH, Black RJ, Macfarlane GJ. Risk of malignancy among patients with rheumatic conditions. International Journal of Cancer. 2000; 88(3):497-502.

Tosovic A, Bondeson A-G, Bondeson L, Ericsson U-B, Malm J, Manjer J. Research article Prospectively measured triiodothyronine levels are positively associated with breast cancer risk in postmenopausal women. 2010

Turner MC, Chen Y, Krewski D, Ghadirian P, Thun MJ, Calle EE. Cancer mortality among US men and women with asthma and hay fever. Am J Epidemiol. 2005; 162(3):212-221. [PubMed: 15987724]

Ukraintseva SV, Arbeev KG, Akushevich I, Kulminski A, Arbeeva L, Culminskaya I, et al. Trade-offs between cancer and other diseases: do they exist and influence longevity? [Research Support, N.I.H., Extramural]. Rejuvenation Res. 2010; 13(4):387-396. [PubMed: 20426618]

Ukraintseva SV, Yashin AI. Individual aging and cancer risk: how are they related. Demographic Research. 2003; 9(8):163-196.

van Heemst D, Mooijaart SP, Beekman M, Schreuder J, de Craen AJM, Brandt BW, et al. Variation in the human TP53 gene affects old age survival and cancer mortality. Experimental gerontology. 2005; 40(1):11-15. [PubMed: 15732191]

Wang F, Gupta S, Holly EA. Diabetes mellitus and pancreatic cancer in a population-based casecontrol study in the San Francisco Bay Area, California. Cancer Epidemiology Biomarkers \& Prevention. 2006; 15(8):1458-1463.

Yang YX, Hennessy S, Lewis JD. Insulin therapy and colorectal cancer risk among type 2 diabetes mellitus patients. Gastroenterology. 2004; 127:1044-1050. [PubMed: 15480982]

Yashin AI, Manton KG, Stallard E. Dependent competing risks: a stochastic process model. Journal of Mathematical Biology. 1986; 24(2):119-140. [PubMed: 3746135]

Yashin A, Akushevich I, Ukraintseva S, Akushevich L, Arbeev K, Kulminski A. Trends in survival and recovery from stroke: evidence from the National Long-Term Care Survey/Medicare data. Stroke. 2010; 41(3):563-565. [PubMed: 20093634]

Yashin AI, Ukraintseva SV, Akushevich IV, Arbeev KG, Kulminski A, Akushevich L. Trade-off between cancer and aging: What role do other diseases play?: Evidence from experimental and human population studies. Mechanisms of Ageing and Development. 2009; 130(1):98-104. [PubMed: 18452970]

Zanetti R, Loria D, Rosso S. Melanoma, Parkinson's disease and levodopa: causal or spurious link? A review of the literature. Melanoma research. 2006; 16(3):201-206. [PubMed: 16718266] 


\section{Highlights}

Mutual dependences in disease risks among US elderly are detected using Medicare data

Cancer risks are decreased for patients with neurogedenerative diseases

Mutually increased risks of goiter and breast cancer were discovered and evaluated

Our data shows that pancreatic cancer and diabetes both increase each other risks 


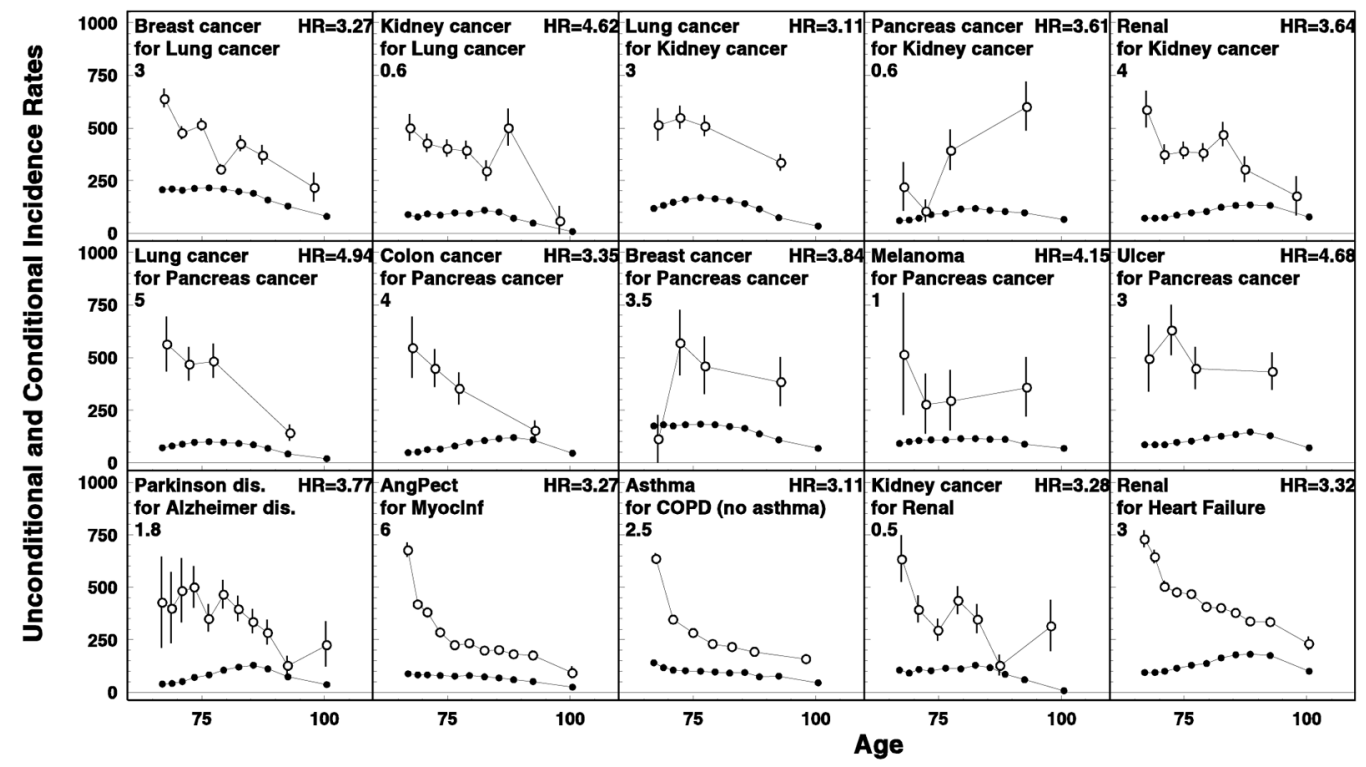

Figure 1.

Direct dependency (i.e., increased risk of later occurring disease) in disease risks when the conditional risk of the later diagnosed diseases was significantly higher $(p<0.001)$ than in general population. Age-specific rates of unconditional (closed circles) and conditional (open circles) rates are shown for diseases in the first lines in the inset in each plot. Conditional rates are calculated for cohorts of individuals earlier diagnosed by another disease (the second lines in each plot). Hazard ratios of the later occurred disease onset for patients with vs. without the pre-existing disease are shown in the right-upper corners. Rescaled factors are shown in the left: the true rate can be calculated by multiplying the values obtained from plot's scale by the rescaled factor. 


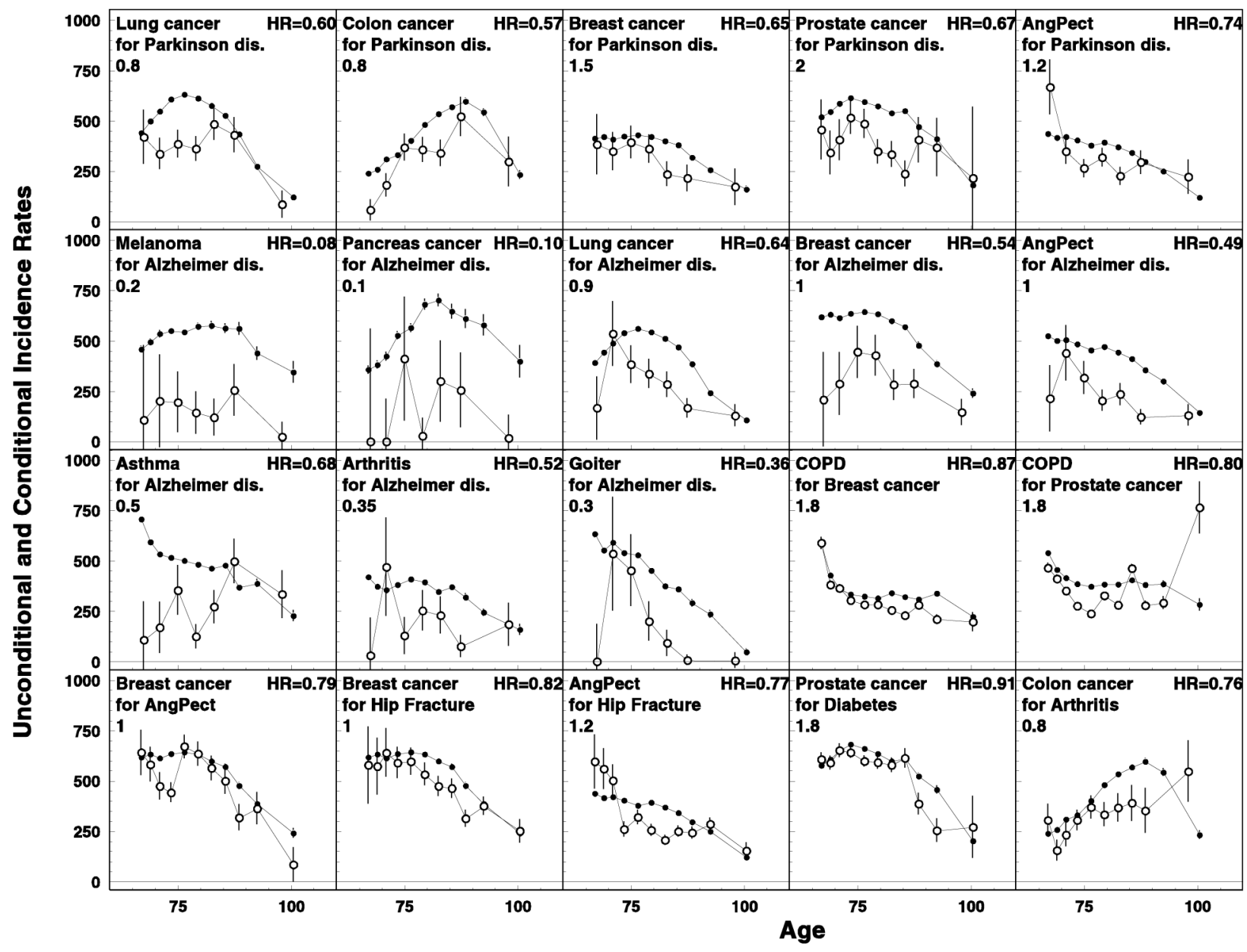

Figure 2.

Inverse effects (or, trade-offs) in the disease risks when the conditional risk of the later occurred disease is lower than in general population. All notations are similar to those for Figure 1. 


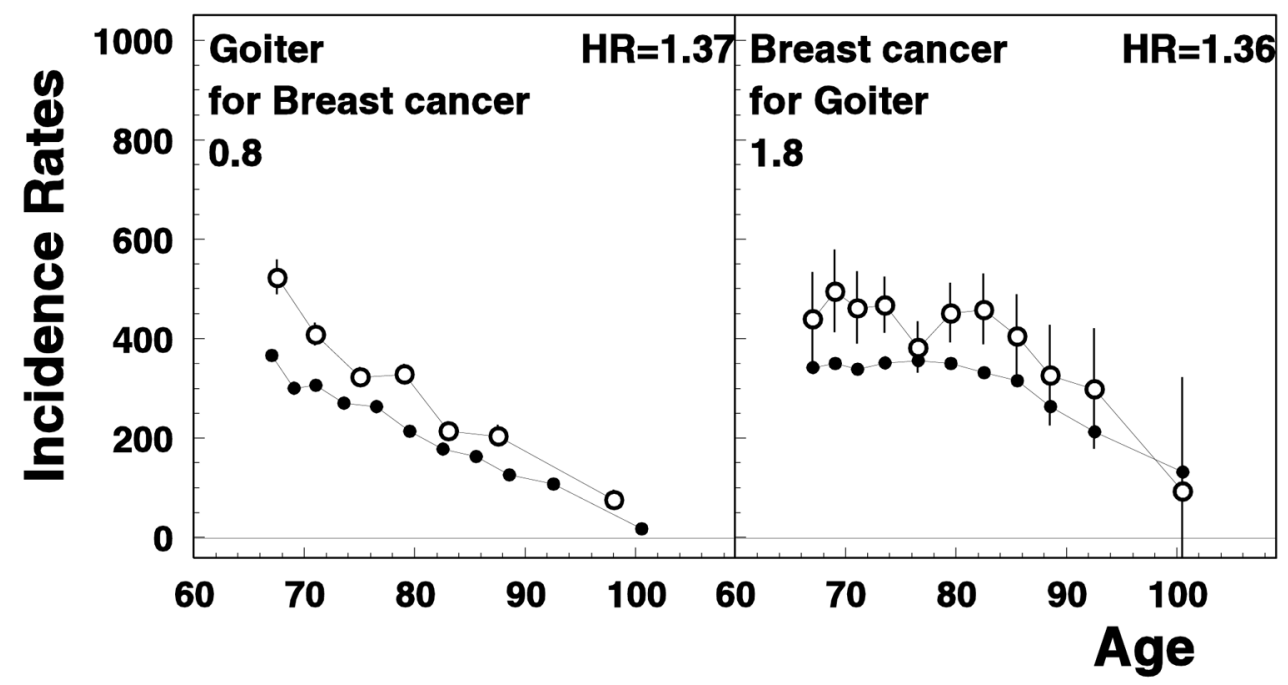

Figure 3.

Age-specific rates of unconditional (closed circles) and conditional (open circles) disease rates for breast cancer and goiter. All notations are similar to those for Figure 1. 


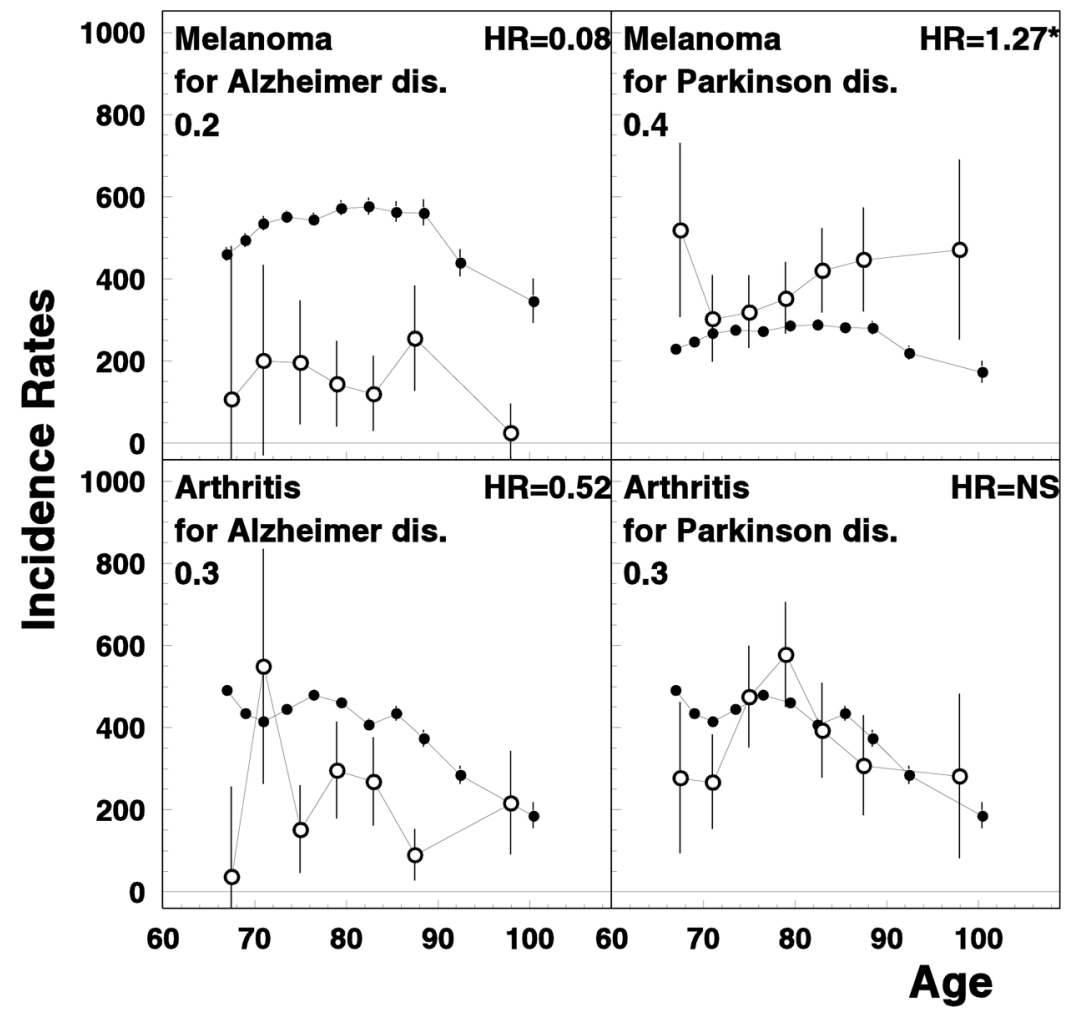

Figure 4.

Age-specific rates of unconditional (closed circles) and conditional (open circles) melanoma and rheumatoid arthritis rates for Alzheimer's disease and Parkinson's disease patients. All notations are similar to those for Figure 1. 


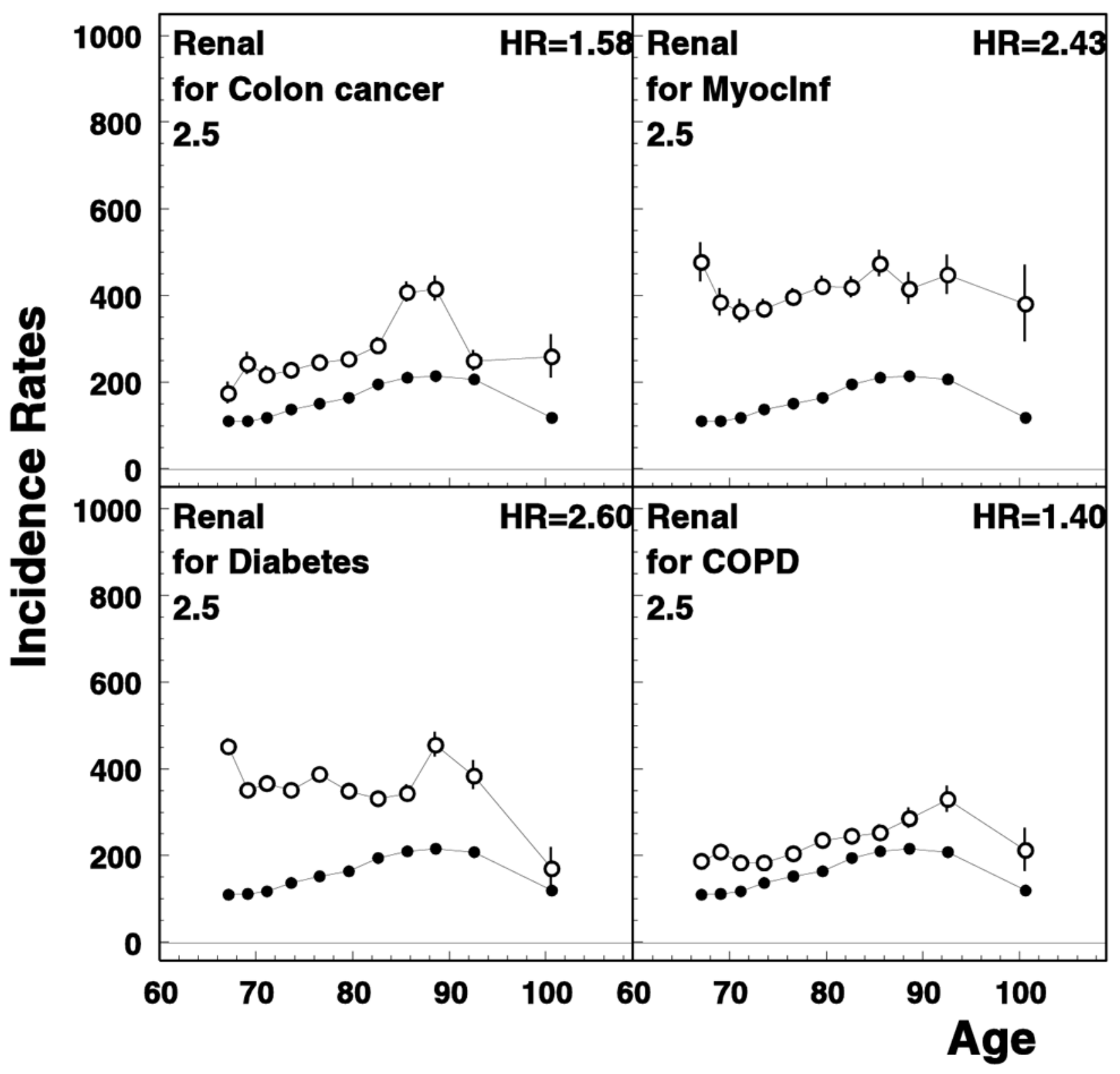

Figure 5.

Age-specific rates of unconditional (closed circles) and conditional (open circles) chronic renal disease rates for patients of selected diseases. All notations are similar to those for Figure 1. 


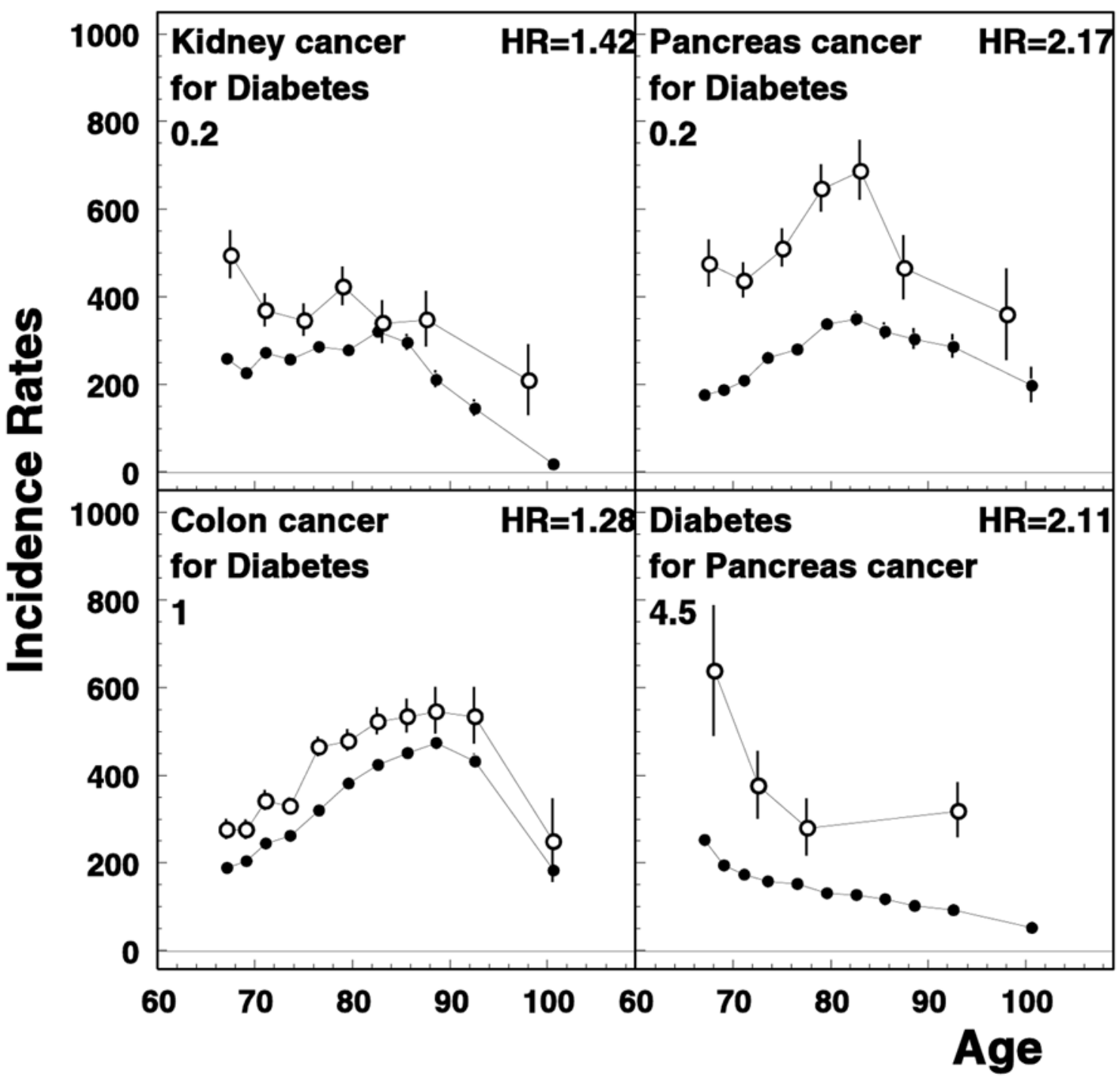

Figure 6.

Selected age-specific rates of unconditional (closed circles) and conditional (open circles) involving diabetes mellitus. All notations are similar to those for Figure 1. 